Old Dominion University

ODU Digital Commons

Bioelectrics Publications

Frank Reidy Research Center for Bioelectrics

2005

\title{
Predicted Properties of Microhollow Cathode Discharges in Xenon
}

J.P. Boeuf

L. C. Pitchford

K.H. Schoenbach

Old Dominion University

Follow this and additional works at: https://digitalcommons.odu.edu/bioelectrics_pubs

Part of the Electrical and Electronics Commons, Elementary Particles and Fields and String Theory Commons, Physical Chemistry Commons, and the Plasma and Beam Physics Commons

\section{Repository Citation}

Boeuf, J. P.; Pitchford, L. C.; and Schoenbach, K. H., "Predicted Properties of Microhollow Cathode Discharges in Xenon" (2005). Bioelectrics Publications. 234.

https://digitalcommons.odu.edu/bioelectrics_pubs/234

\section{Original Publication Citation}

Boeuf, J. P., Pitchford, L. C., \& Schoenbach, K. H. (2005). Predicted properties of microhollow cathode discharges in xenon. Applied Physics Letters, 86(7), 071501. doi:10.1063/1.1862781

This Article is brought to you for free and open access by the Frank Reidy Research Center for Bioelectrics at ODU Digital Commons. It has been accepted for inclusion in Bioelectrics Publications by an authorized administrator of ODU Digital Commons. For more information, please contact digitalcommons@odu.edu. 


\title{
Predicted properties of microhollow cathode discharges in xenon
}

\author{
J. P. Boeuf and L. C. Pitchford ${ }^{\text {a) }}$ \\ Centre de Physique des Plasmas et Applications de Toulouse (CPAT), CNRS UMR 5002, \\ 31062 Toulouse, France
}

\section{K. H. Schoenbach}

Center for Bioelectrics, Old Dominion University, Norfolk, Virginia 23529

(Received 26 August 2004; accepted 14 December 2004; published online 7 February 2005)

\begin{abstract}
A fluid model has been developed and used to help clarify the physical mechanisms occurring in microhollow cathode discharges (MHCD). Calculated current-voltage $(I-V)$ characteristics and gas temperatures in xenon at 100 Torr are presented. Consistent with previous experimental results in similar conditions, we find a voltage maximum in the $I-V$ characteristic. We show that this structure reflects a transition between a low-current, abnormal discharge localized inside the cylindrical hollow cathode to a higher-current, normal glow discharge sustained by electron emission from the outer surface of the cathode. This transition, due to the geometry of the device, is a factor contributing to the well-known stability of MHCDs. (C) 2005 American Institute of Physics.
\end{abstract}

[DOI: $10.1063 / 1.1862781]$

Considerable effort has been devoted over the past ten years to the development of stable, high-pressure, nonthermal plasma sources. One discharge concept that has proven to be stable at atmospheric pressure and up to a power density of some $100 \mathrm{~kW} / \mathrm{cm}^{3}$ is the microhollow cathode discharge $^{1}$ (MHCD) developed by Schoenbach and coworkers. These discharges are created by applying a voltage between two electrodes in a geometry consisting of a cathode/dielectric/anode sandwich through which a hole is drilled. The thickness of the dielectric separating the electrodes and the diameter of the hole are both on the order of one to several hundred microns. When arranged in an array, a large number of MHCDs operating in an abnormal glow mode can generate a high-pressure, dc, "surface plasma", with electron densities ${ }^{3}$ up to $10^{15} \mathrm{~cm}^{-3}$. A high-pressure $\mathrm{dc},{ }^{4}$ or pulsed," "volume plasma" can be generated with a third, planar electrode, located about a centimeter from the MHCD array. In this configuration the MHCD is used as an electron source for a larger volume discharge. Other high-pressure plasma sources based on this idea are described in a review paper by Kunhardt. ${ }^{6}$

We present here results from a simple model of a single MHCD. The aim of our work is to provide estimates of the main discharge and plasma parameters and to help understand the basic mechanisms governing the MHCD devices. The model used is based on solutions of fluid equations in the drift-diffusion approximation for the electron and ion transport coupled with Poisson's equation. Dielectric boundary conditions are imposed on the open part of the computational domain to fix the boundary condition on the potential. This boundary is taken to be far enough from the active discharge region that it has no influence on the results. The ionization rate coefficient appearing in the electron and ion source terms is supposed to depend on the mean electron energy, ${ }^{7}$ and an equation for the electron energy is added to the system of equations to be solved.

Gas heating leads to a decrease in the local neutral density, and this can have important consequences on electron

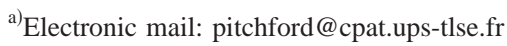

energy deposition in the sheath region and therefore on the sheath structure itself. Gas heating in a MHCD in xenon is due mainly to power deposition by ions in the sheath region (elastic and charge exchange collisions), but it is difficult to quantify the gas heating source term because a large part of the ion energy can be released on the cathode (by ions or fast neutrals) instead of in the gas. ${ }^{8}$ In this first approach, we consider that a given, fixed fraction of the ion heating is converted locally into gas heating, the rest being deposited on the cathode which is fixed at $300 \mathrm{~K}$. Using this as a source term, the gas temperature is obtained from the heat equation. Electron heating of the gas is taken into account but is negligible for our conditions, and gas flow is not taken into account.

To estimate the contribution of the xenon excited species to the overall ionization balance and the contribution of molecular ion formation and recombination to the overall electron loss, a very simple kinetic model is used. The species considered in the simulation are electrons, atomic ions, molecular ions, and metastable atoms. The model of the xenon metastable density is based on Ref. 9, but simplified by assuming that the ratio of resonance to metastable atoms is constant.

The geometry of the MHCD used in the model calculations and its dimensions are given in Fig. 1. This geometry is similar to that used in the experimental work of Schoenbach et al. ${ }^{1}$ The calculated current-voltage characteristic for a xenon discharge in this geometry at 100 Torr is shown by the solid points in Fig. 2. The following values were used for the

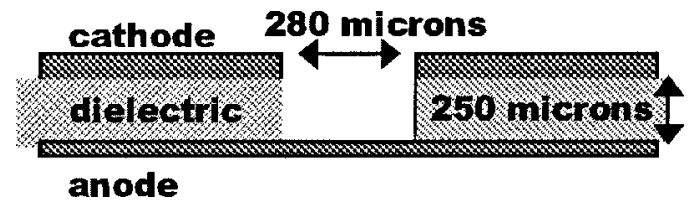

FIG. 1. Discharge geometry. The hollow cathode hole diameter is $280 \mu \mathrm{m}$, the electrodes are spaced by $250 \mu \mathrm{m}$, and each is $100 \mu \mathrm{m}$ thick. The only information about the electrode and insulator material needed for the calculations are the values of the secondary-electron emission coefficients and the surface temperature. 


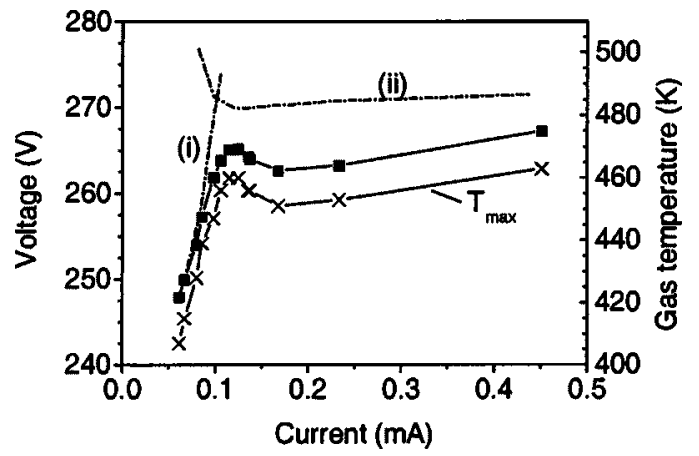

FIG. 2. Calculated $I-V$ characteristic $(\square)$ and peak gas temperature $(\times)$ vs current. The dashed lines (i) and (ii) are characteristics calculated for discharges sustained by electron emission from inside the hollow cathode or from the outer surface of the cathode, respectively.

parameters: secondary electron emission coefficients of 0.002 and 0.005 due ion bombardment, and metastable bombardment, respectively; the fraction of ion power deposited locally in gas heating of $25 \%$; a surface temperature of 300 $\mathrm{K}$; and the ratio of resonance to metastable density of 0.1 . While these values are estimates only, our quantitative predictions are not very dependent on these values, at least at 100 Torr.

The shape of the calculated characteristic is similar to those measured by Schoenbach et al. ${ }^{1}$ in argon and in xenon. ${ }^{10}$ There is a steep increase in voltage at low current, which is followed for increasing current by a region of negative slope and then a plateau. The region of negative slope was attributed by Schoenbach et al. ${ }^{1}$ to the onset of the classical hollow cathode effect. This interpretation was based in part on the calculations of Fiala et al. ${ }^{11}$ in argon HCDs, but for lower values of pd (produce of gas pressure and cathode hole diameter) and where a marked decrease in the voltage was observed to be associated with efficient ionization due to electrons oscillating between opposite sheaths inside the hollow cathode; i.e., ionization due to pendulum electrons or the classical hollow cathode effect.

For the conditions of the calculations here, the structure in the $I-V$ characteristic is not due to pendulum electrons because the electron energy-loss mean free path is too short to allow electrons emitted from one part of the cathode to reach the opposite sheath with enough energy to ionize. Taking into account a decrease in gas density (i.e., an increase in mean free path) with increasing gas temperature, we estimate that gas temperatures greater than $1000 \mathrm{~K}$ at 100 Torr would be needed before pendulum electrons contribute to ionization.

The voltage maximum observed in our calculations is associated with a transition from a glow discharge localized inside the hollow cathode $(<0.12 \mathrm{~mA})$ to a glow discharge spreading along the outer cathode surface $(>0.12 \mathrm{~mA})$. Thus, the steep slope of the $I-V$ characteristic at low current corresponds to an abnormal glow discharge inside the hollow cathode; increasing current is achieved by increasing the current density. Above $0.12 \mathrm{~mA}$, enough ions and metastables from the hollow cathode discharge reach the outer surface of the cathode to initiate a discharge between the outer cathode surface and the anode. Once initiated, this discharge is sustained by ions and metastables created mainly in the volume outside the hollow cathode. This second discharge is in parallel with the first discharge localized inside the hollow cath-

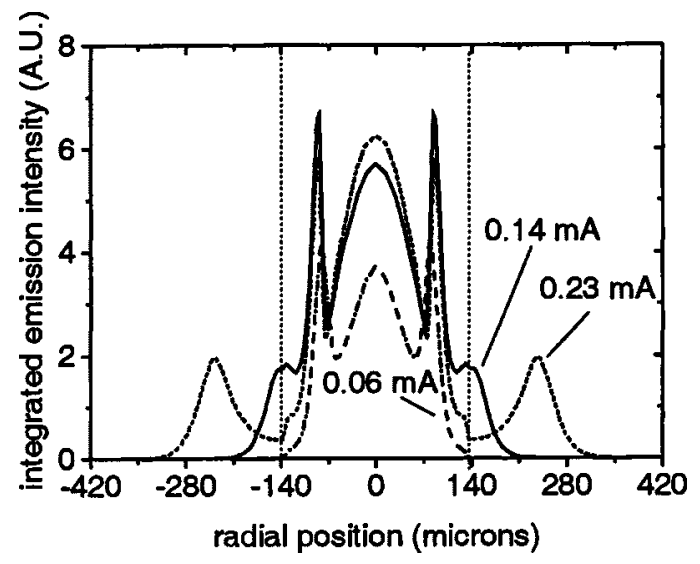

FIG. 3. Emission intensity integrated along the line of sight vs radial distance from the axis for three points along the baseline characteristic as indicated. The vertical lines show the position of the hollow cathode walls.

ode, and the $I-V$ characteristic above $0.12 \mathrm{~mA}$ reflects the behavior of the normal glow discharge along the outer cathode surface. It is interesting to compare the MHCD characteristic with calculated characteristics of independent discharges inside the hollow cathode [curve (i) in Fig. 2], and between the outer surface of the cathode and the anode in curve (ii). Curves (i) and (ii) were calculated by setting secondary-electron emission coefficients to zero on the outer cathode face or inside the hollow cathode, respectively.

In Fig. 3 we have plotted the line-of-sight emission intensity, calculated assuming each excitation event leads locally to the emission of one photon, for three different points along the $I-V$ characteristic. Quantitatively, these results compare well with the experiments of Schoenbach et al. ${ }^{1}$ At low current, all excitation is well-localized inside the hollow cathode and, abruptly above $0.12 \mathrm{~mA}$, excitation is observed at radial positions greater than the hollow cathode radius. The on-axis peaks are the result of integrating through the positive column, and these are relatively more important with increasing current. The sharp, off-axis peaks in the emission intensity inside the hollow cathode occur near the edges of the sheaths. (See also Ref. 12 for relevant experimental results at lower pressures.)

Returning now to the plot of peak gas temperature $T_{\max }$ versus current in Fig. 2, we see that $T_{\max }$ increases rapidly in the abnormal discharge regime below $0.12 \mathrm{~mA}$, where the ion current density at the inner walls of the hollow cathode is increasing. After the discharge has spread to the outer cathode surface, $T_{\max }$ tends to saturate, like the discharge voltage. The maximum gas temperature is always inside the hollow cathode. This saturation in the gas temperature is probably an important factor contributing to the stability of MHCDs.

The peak plasma density at $0.12 \mathrm{~mA}$ is about 3 $\times 10^{13} \mathrm{~cm}^{-3}$. On axis and inside the hollow cathode, $30 \%$ of the ions are molecular ions. The maximum metastable density is about $3 \times 10^{14} \mathrm{~cm}^{-3}$ and its peak is off-axis near the sheath edges. Two-step ionization of the xenon metastables represents only about $10 \%$ to $15 \%$ of the total ionization.

Other calculations, not illustrated here, were made to estimate the effects of uncertainites in the gas heating source term and to better understand the effects of metastables. In all cases, we find the same general behavior of the MHCD, but the detailed shape of the $I-V$ curve depends on the exact values of the parameters used in the calculations. For example, the voltage maximum in the $I-V$ characteristic is 
somewhat less pronounced and is shifted towards lower currents when a higher fraction of the ion power goes into gas heating. The peak gas temperature $T_{\max }$ is always inside the hollow cathode and, for a given current, it is almost proportional to the voltage. $T_{\max }$ increases from $460 \mathrm{~K}$ to almost $700 \mathrm{~K}$ at $0.12 \mathrm{~mA}$ as the fractional ion power going into gas heating is increased from $25 \%$ to $100 \%$. Note that the gas temperature estimated in MHCDs in argon at 100 Torr by Penache et al. ${ }^{13}$ at $0.5 \mathrm{~mA}(200 \mathrm{~V})$ was about $500 \mathrm{~K}$, and we would expect the same discharge in xenon to be somewhat hotter because of lower thermal conductivity of xenon.

When metastable-induced secondary-electron emission from the cathode is set to zero, the $I-V$ characteristic is shifted up to higher voltages, but the shape remains essentially the same. There is no voltage maximum present in calculations without metastables, but there is a sudden decrease in the slope of the $I-V$ characteristic when the discharge spreads to the outer surface of the cathode.

Results of calculations for other gas pressures are similar to those presented earlier. One difference with experiment is that our model predicts an increase in voltage with gas pressure, whereas the opposite is found in experiments. ${ }^{10}$ A process not accounted for in our model that could be important in this comparison is photoemission.

To summarize, although the model used here is approximate and more work needs to be done to improve the quantitative predictions, it is nevertheless useful for understanding the behavior of MHCDs. Our results show that with increasing current in MHCDs there is a transition from abnormal discharge inside the hollow cathode to a normal glow discharge where much of the current is drawn through the outer surface of the cathode. After the transition, the power deposited in the hollow cathode region does not increase significantly when the current increases, provided that the discharge can continue to spread on the outer surface of the cathode. This has implications for the discharge stability because the gas temperature (and electric field at the cathode surface-not shown here) tend to saturate with increasing current as long as there is available cathode surface for the discharge to spread.

This work was partially suppored by US Air Force through EOARD in London and by the National Science Foundation (CTS-0078618).

${ }^{1}$ K. H. Schoenbach, A. El-Habachi, W. Shi, and M. Ciocca, Plasma Sources Sci. Technol. 6, 468 (1997).

${ }^{2}$ K. H. Schoenbach, M. Moselhy, W. Shi, and R. Bentley, J. Vac. Sci. Technol. A 21, 1 (2003).

${ }^{3}$ M. Moselhy, I. Petzenhauser, K. Frank, and K. H. Schoenbach, J. Phys. D 36, 2922 (2003)

${ }^{4}$ R. H. Stark and K. H. Schoenbach, J. Appl. Phys. 85, 2075 (1999)

${ }^{5}$ R. H. Stark and K. H. Schoenbach, J. Appl. Phys. 89, 3568 (2001)

${ }^{6}$ E. E. Kunhardt, IEEE Trans. Plasma Sci. 28, 189 (2000)

${ }^{7}$ J.-P. Boeuf and L. C. Pitchford, Phys. Rev. E 51, 1376 (1995).

${ }^{8}$ I. Revel, L. C. Pitchford, and J.-P. Boeuf, J. Appl. Phys. 88, 2234 (2000).

${ }^{9}$ L. C. Pitchford, J. Kang, C. Punset, and J.-P. Boeuf, J. Appl. Phys. 92, 6990 (2002).

${ }^{10}$ M. M. Moselhy and K. H. Schoenbach (private communication).

${ }^{11}$ A. Fiala, L. C. Pitchford, and J.-P. Boeuf, Proceedings of the XXII International Conference on Phenomena in Ionized Gases, Hoboken, NJ, 1995, Contr. Papers 4, p. 191.

${ }^{12}$ Th. Callegari, F. Gegot, L. C. Pitchford, J. Galy, J.-P. Boeuf, IEEE Trans. Plasma Sci. (in press).

${ }^{13}$ C. Penache, M. Miclea, A. Bräuning-Demian, O. Hohn, S. Schössler, T. Jahnke, K. Neimax, and H. Schmidt-Böcking, IEEE Trans. Plasma Sci. 11, 476 (2002). 